\title{
Polydatin possesses notable anti-osteoporotic activity via regulation of OPG, RANKL and $\beta$-catenin
}

\author{
QI-LIN ZHOU, RU-ZI QIN, YAN-XIN YANG, KUN-BING HUANG and XIAN-WEN YANG \\ Department of Internal Medicine, Guangdong Province Corps Hospital, \\ Chinese People's Armed Police Forces, Guangzhou, Guangdong 510507, P.R. China
}

Received July 20, 2015; Accepted June 6, 2016

DOI: $10.3892 / \mathrm{mmr} .2016 .5432$

\begin{abstract}
This study was designed to investigate the anti-osteoporotic activity of polydatin and its possible underlying mechanism. Osteoporosis was induced in mice by ovariectomy (OVX) and the mice were divided into 5 groups: An OVX only group, polydatin groups $(10,20$ and $40 \mathrm{mg} / \mathrm{kg}$ ) and a sham group (n=10/group). After 12 weeks of treatment, body weight, uterine index and the dry weight of thigh-bones were recorded. In addition, the serum calcium, serum phosphorus, alkaline phosphatase (ALP) and osteoprotegerin (OPG) levels were also determined. Western blot analysis was then conducted to investigate the possible mechanism underlying the effect of polydatin via determining the expression of OPG, receptor activators of nuclear factor- $\kappa \mathrm{B}$ ligand (RANKL) and $\beta$-catenin in the ST2 cell line. The results indicated that intraperitoneal injection of polydatin (10,20 and $40 \mathrm{mg} / \mathrm{kg} /$ day) decreased body weight, and increased uterine index and dry weights of thigh-bones of ovariectomized mice $(\mathrm{P}<0.05)$, and polydatin also significantly increased the serum calcium, phosphorus, ALP and OPG of ovariectomized mice $(\mathrm{P}<0.05)$. Results of western blot analysis showed that polydatin upregulated the ratio of OPG/RANKL $(\mathrm{P}<0.05)$ and $\beta$-catenin protein in ST2 cells. In conclusion, the results demonstrated that polydatin exhibits anti-osteoporotic activity via regulating osteoprotegerin, RANKL and $\beta$-catenin.
\end{abstract}

\section{Introduction}

Osteoporosis is a progressive bone disease characterized by low bone mass, microarchitectural deterioration of bone tissue and a high risk of fractures $(1,2)$. In addition, osteoporosis

Correspondence to: Dr Qi-Lin Zhou, Department of Internal Medicine, Guangdong Province Corps Hospital, Chinese People's Armed Police Forces, 268 Yanling Road, Tianhe, Guangzhou, Guangdong 510507, P.R. China

E-mail: qiwzhouapf@163.com

Key words: polydatin, anti-osteoporotic activity, ST2 cells, osteoprotegerin, receptor activators of nuclear factor- $\kappa \mathrm{B}$ ligand, $\beta$-catenin can easily result in serious bone fragility and susceptibility to fracture (3). It was estimated that over 200 million individuals has osteoporosis worldwide in 2013 and $30 \%$ of the postmenopausal women in the USA and Europe suffer with osteoporosis $(4,5)$. Currently, estrogen is one of the most commonly used treatment strategies for treating postmenopausal osteoporosis (6). However, studies have demonstrated that estrogen treatment could result in serious adverse effects, such as endometrial carcinoma, breast cancer and cardiovascular disease (4,7). It is therefore key to identify novel strategies with low toxicity for treating osteoporosis.

Polydatin (3,4',5-trihydroxystilbene-3- $\beta$-D-glucoside), predominantly isolated from the roots of Polygonum cuspidatum Sieb, is a known natural stilbenes compound with wide pharmacological activity (8). Previous studies have demonstrated that polydatin possesses notable anti-inflammatory, anti-oxidant, anti-shock, anti-asthmatic and anti-hypertrophic effects (8-13). Postmenopausal osteoporosis is associated with ovarian hormone deficiency and is a common reason for age-related bone loss (14). The ovariectomized rat model is a commonly used osteoporosis animal model due to its similarities in etiology and pathology to postmenopausal osteoporosis. Blood calcium and phosphorus are two important elements that are key for the integrity and remodeling of bone; alkaline phosphatase (ALP) is a crucial enzyme for bone remodeling; and receptor activators of nuclear factor- $\kappa \mathrm{B}$ ligand/osteoprotegerin (RANKL/OPG) is reported to be important for the formation and differentiation osteoclasts. Thus, these indicators are commonly used to investigate osteoporosis and were analyzed in this study $(2-4,14)$. The present study was designed to investigate the anti-osteoporotic activity of polydatin on postmenopausal osteoporosis using the ovariectomized rat model, and to determine its related molecular mechanisms.

\section{Materials and methods}

Chemicals. Polydatin was purchased from Shanghai Tauto Biotech Co., Ltd. (Shanghai, China), and its purity was $>98 \%$. $\alpha$-modified minimum essential medium ( $\alpha$-MEM) and fetal bovine serum (FBS) were purchased from Gibco, Thermo Fisher Scientific, Inc. (Waltham, MA, USA). Dimethyl sulfoxide (DMSO) and serum OPG enzyme-linked immunosorbent assay (ELISA) kits were purchased from Sigma-Aldrich (St. Louis, MO, USA). Rabbit anti-RANKL (cat. no. BA1323 
1:500) and OPG (cat no. BA1475 1:500) polyclonal antibodies were purchased from Wuhan Boster Bio-engineering Co., Ltd. (Wuhan, China). Rabbit anti- $\beta$-catenin (cat. no. ab6302; $1: 2,000$ ), anti-histone (cat. no. ab1791; $1: 2,000)$ and anti- $\beta$-actin (cat. no. ab5694; 1:2,000) polyclonal antibodies were purchased from the Abcam (Cambridge, MA, USA). Bicinchoninic acid (BCA) protein assay reagents and western blot \& IP cell lysis buffer kits were purchased from Beyotime Co. (Hangzhou, China). All other regents used in this study were of analytical grade.

Animals. Animal protocols were established according to the generally accepted international guidelines, and approved by the Animal Care and Use Committee of the Guangdong Province Corps Hospital (Chinese People's Armed Police Forces, Guangzhou, China; approval no. 20141109_01\#). Female ICR mice (age, 3 months; $n=10 /$ group; weight, $20 \pm 2 \mathrm{~g}$ ) used in the present study were purchased form the Shanghai Laboratory Animal Center (Shanghai, China). All animals were housed at $21 \pm 1^{\circ} \mathrm{C}$ and $50-60 \%$ humidity under a $12 \mathrm{~h}$ light/dark cycle and had free access to standard pellet diet and tap water.

Toxicity tests. The 80 ICR mice were random divided into 8 groups $(\mathrm{n}=10)$. Mice of groups 1-7 were administered 2.5, $5,10,20,40,80$ or $100 \mathrm{mg} / \mathrm{kg}$ polydatin by intraperitoneal injection (i.p.), respectively. Group 8 was administered normal saline $(10 \mathrm{ml} / \mathrm{kg}$, i.p.). The mortality rate of the mice in each group was observed during a $24 \mathrm{~h}$ period. Notably, neither death nor any abnormal neurobehavior were observed, and thus the lethal dose (LD) ${ }_{50}$ of polydatin was not obtained.

Preparation of ovariectomy mice and experimental protocol. Osteoporosis was induced by ovariectomy (OVX) surgery according to the method described by Kalu (14). After general anesthetic with $50 \mathrm{mg} / \mathrm{kg}$, i.p. sodium pentobarbital, an incision was made through the back and bilateral ovaries were resected. Mice undergoing sham surgery mice underwent the same surgical procedures without ovary resection.

The prepared OVX mice were randomly divided into an OVX group, 3 polydatin treatment groups $(10,20$ and $40 \mathrm{mg} / \mathrm{kg})$ and sham surgery group. The polydatin treatment lasted 12 weeks; following treatment body weights of all the mice were recorded. Subsequently, mice were sacrificed via cervical dislocation under sodium pentobarbital $(50 \mathrm{mg} / \mathrm{kg}$, i.p.) anesthetic. Blood samples $(\sim 1 \mathrm{ml})$ were collected and centrifuged at $4^{\circ} \mathrm{C}$ at $3,000 \mathrm{x} \mathrm{g}$ for $10 \mathrm{~min}$ to obtain serum for further experiments. Furthermore, the uterus were collected and weighed immediately to calculate the uterine index, and the thigh-bones of mice were collected to investigate the dry weights.

Determination of serum ALP, calcium and phosphorus. The serum ALP, calcium and phosphorus levels were determined using the automatic biochemistry analyzer (Beckman Coulter AU5800, Brea, CA, USA).

Determination of $O P G$ in the serum. The serum OPG level was determined using a commercial ELISA kit, according to the manufacturer's instructions and was measured using a microplate reader (Multiskan FC, Thermo Fisher Scientific, Inc.) at $450 \mathrm{~nm}$.
Cell culture. The ST2 mouse bone marrow stromal cell line was purchased from the American Type Culture Collection (Mannasas, VA, USA). The cells were cultured in $\alpha$-MEM medium which was supplemented with $10 \% \mathrm{FBS}$, and the cells were cultured at $37^{\circ} \mathrm{C}$ in $5 \% \mathrm{CO}_{2} / 95 \%$ air.

Western blotting. ST2 cells were treated with or without polydatin $(10,20$ and $40 \mu \mathrm{g} / \mathrm{ml})$ for $48 \mathrm{~h}$, and then were harvested. Total proteins of cells were extracted using the western blot and IP cell lysis buffer kit. After quantification of total protein using the BCA protein assay reagent, $40 \mu \mathrm{g}$ protein was separated by $10 \%$ sodium dodecyl sulfate-polyacrylamide gel electrophoresis, blotted on polyvinylidene difluoride membranes, and probed with various primary antibodies, and subsequently with horseradish-peroxidase-conjugated secondary antibody (Wuhan Boster Bio-engineering Co., Ltd.) and detected by chemiluminescence with BeyoECL Plus reagents (Beyotime Institute of Biotechnology, Jiangsu, China). To measure protein loading, antibodies directed against $\beta$-actin/histone were used.

Statistical analysis. Data are expressed as the mean \pm standard deviation. Statistical analyses were performed using one-way analysis of variance followed by Dunnett's multiple comparisons test using SPSS software 18.0 (SPSS Inc., SPSS Inc., Chicago, IL, USA). $\mathrm{P}<0.05$ was considered to indicate a statistically significant difference.

\section{Results}

Effects of polydatin on body weight and uterine index of ovariectomized mice. As shown in Fig. 1, after 12 weeks of treatment of polydatin, the ovariectomized mice had a significantly increased body weight compared with the sham mice $(\mathrm{P}<0.01)$. Notably, the increased weight of ovariectomized mice was reversed following treatment with polydatin. Treatment of polydatin at doses of 10,20 and $40 \mathrm{mg} / \mathrm{kg}$ /day significantly prevented the weight gain observed in ovariectomized mice $(\mathrm{P}<0.05, \mathrm{P}<0.01$ and $\mathrm{P}<0.01$, respectively). Furthermore, this occurred in a dose-dependent manner.

The uterine index of the mice was decreased following OVX surgery compared with that in the sham mice $(\mathrm{P}<0.01)$. However, polydatin at doses of 20 and $40 \mathrm{mg} / \mathrm{kg} /$ day increased the uterine index compared with mice in the OVX group $(\mathrm{P}<0.01)$ (Fig. 2).

Effects of polydatin on serum calcium and phosphorus levels in the ovariectomized mice. As shown in Fig. 3, following OVX, the serum calcium and phosphorus levels of the mice in the OVX group were decreased compared with that in the sham mice $(\mathrm{P}<0.01)$. Notably, it was demonstrated that treatment with polydatin $(10,20$ and $40 \mathrm{mg} / \mathrm{kg} /$ day $)$ increased calcium $(\mathrm{P}<0.05)$ and phosphorus levels in the serum $(\mathrm{P}<0.05$, $\mathrm{P}<0.01, \mathrm{P}<0.01$, respectively) compared with the OVX group. This occurred in a dose-dependent manner.

Effects of polydatin on ALP and OPG of ovariectomized mice. As shown in Fig. 4, ALP levels of ovariectomized mice were increased following OVX surgery. Furthermore, polydatin at doses of 10,20 and $40 \mathrm{mg} / \mathrm{kg} /$ day significantly decreased the 


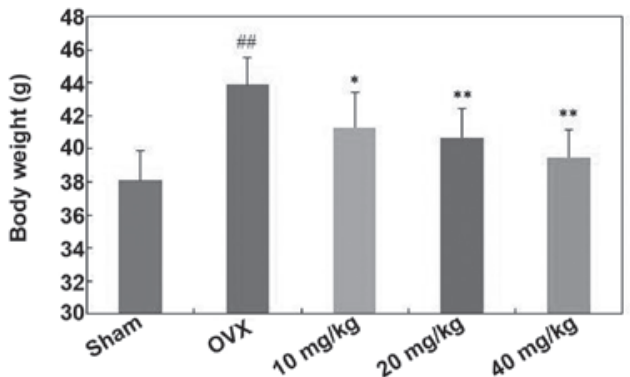

Figure 1. Effects of polydatin on the body weight of ovariectomized mice. Data are presented as the mean \pm standard deviation $(n=10)$. Polydatin $(10,20$ and $40 \mathrm{mg} / \mathrm{kg}$ ) was administered intraperitoneally. ${ }^{\# \#} \mathrm{P}<0.01$, compared with the sham group; ${ }^{*} \mathrm{P}<0.05,{ }^{* *} \mathrm{P}<0.01$, compared with the OVX group. OVX, ovariectomy.

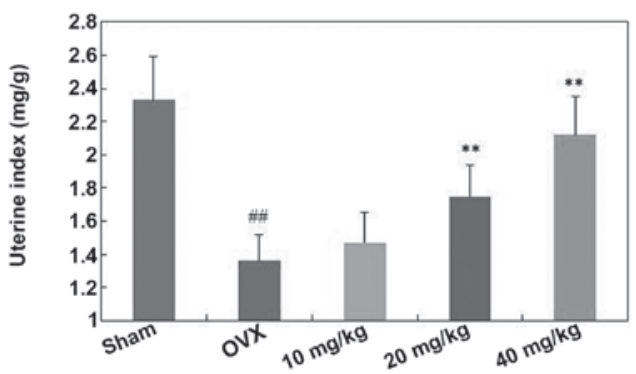

Figure 2. Effects of polydatin on the uterine index of ovariectomized mice. Data are presented as the mean \pm standard deviation $(\mathrm{n}=10)$. Polydatin $(10,20$ and $40 \mathrm{mg} / \mathrm{kg}$ ) was administered intraperitoneally. ${ }^{\# \#} \mathrm{P}<0.01$, compared with the sham group; ${ }^{* *} \mathrm{P}<0.01$, compared with the OVX group. OVX, ovariectomy.
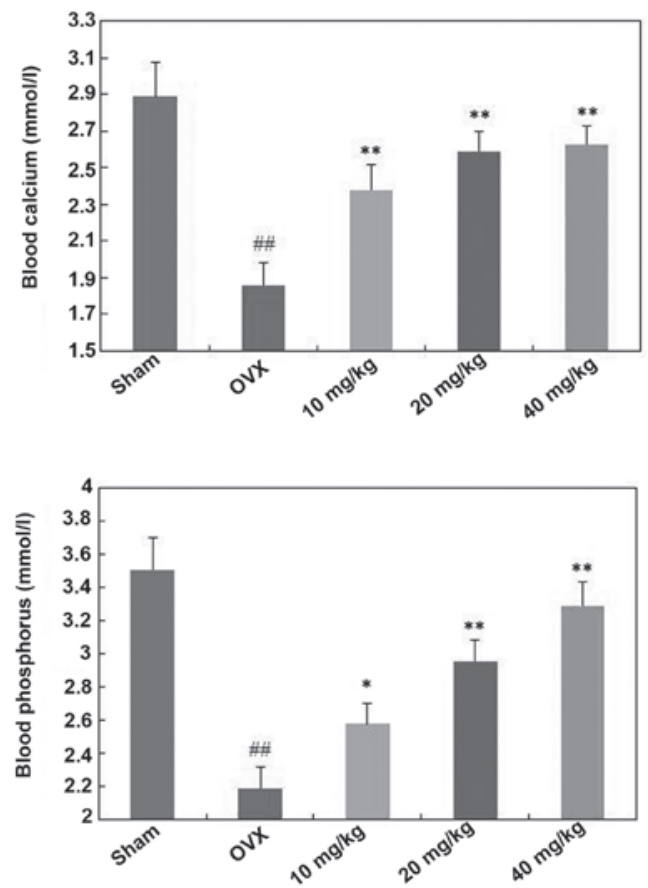

Figure 3. Effects of polydatin on calcium and phosphorus levels of ovariectomized mice. Data are presented as the mean \pm standard deviation $(\mathrm{n}=10)$. Polydatin $(10,20$ and $40 \mathrm{mg} / \mathrm{kg})$ was administered intraperitoneally. ${ }^{\# \prime} \mathrm{P}<0.01$, compared with the sham group; ${ }^{*} \mathrm{P}<0.05$ and ${ }^{* *} \mathrm{P}<0.01$, compared with the OVX group. OVX, ovariectomy.

ALP level compared with the OVX group $(\mathrm{P}<0.05, \mathrm{P}<0.01$ and $\mathrm{P}<0.01$, respectively) in a dose-dependent manner.
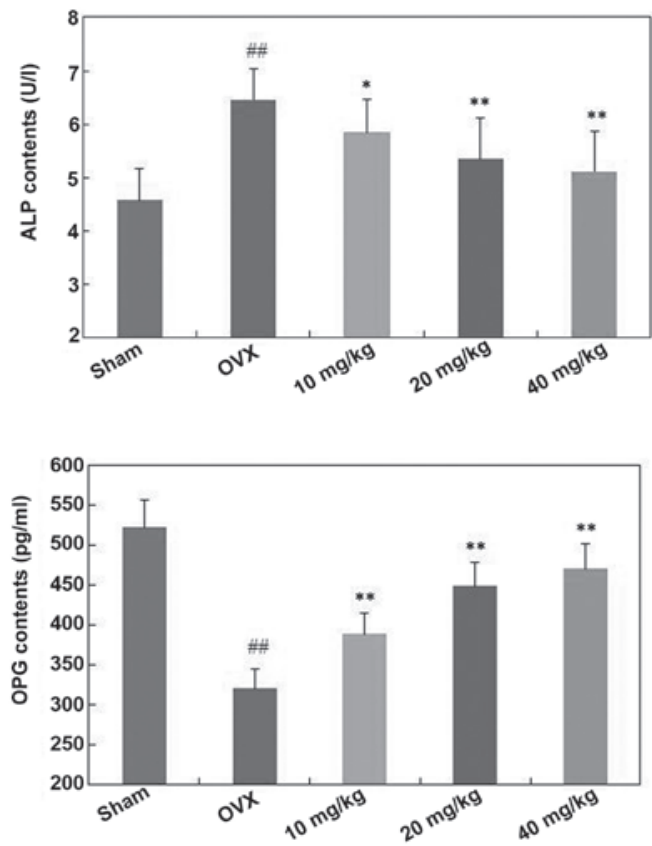

Figure 4. Effects of polydatin on ALP and OPG levels in ovariectomized mice. Data are presented as the mean \pm standard deviation $(n=10)$. Polydatin $(10,20$ and $40 \mathrm{mg} / \mathrm{kg})$ was administered intraperitoneally. ${ }^{\# \#} \mathrm{P}<0.01$, compared with the sham group; ${ }^{*} \mathrm{P}<0.05,{ }^{* *} \mathrm{P}<0.01$, compared with the OVX group. ALP, alkaline phosphatase; OPG, osteoprotegerin; OVX, ovariectomy.

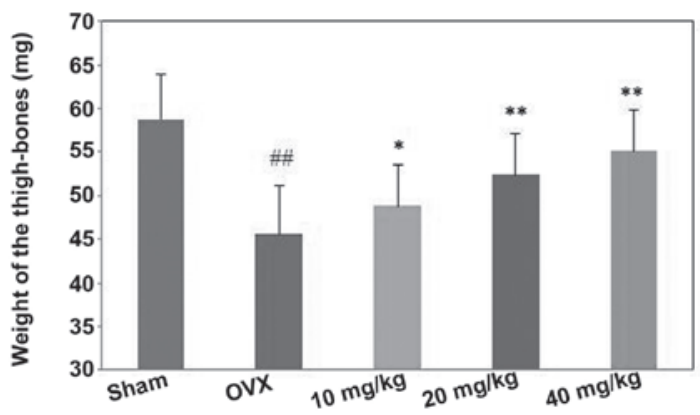

Figure 5. Effects of polydatin on thigh-bone weight of ovariectomized mice. Data are presented as the mean \pm standard deviation $(n=10)$. Polydatin $(10,20$ and $40 \mathrm{mg} / \mathrm{kg}$ ) was administered intraperitoneally. ${ }^{\# \#} \mathrm{P}<0.01$, compared with the sham group; ${ }^{\mathrm{P}} \mathrm{P}<0.05,{ }^{* *} \mathrm{P}<0.01$, compared with the OVX group. OVX, ovariectomy.

Conversely, the OPG levels in the mice of the OVX group were significantly decreased compared with that in the sham mice $(\mathrm{P}<0.01)$ (Fig. 4). Treatment with polydatin $(10,20$ and $40 \mathrm{mg} / \mathrm{kg} / \mathrm{day}$ ) increased the OPG levels in the serum compared with the OVX group $(\mathrm{P}<0.01)$, in a dose-dependent manner.

Effects of polydatin on thigh-bone weight of OVX mice. As shown in Fig. 5, thigh-bone weight of ovariectomized mice decreased following removal of the ovaries compared with that in the sham group $(\mathrm{P}<0.01)$. However, treatment with polydatin $(10,20$ and $40 \mathrm{mg} / \mathrm{kg})$ resulted in a significant elevation in thigh-bone weight compared with that in the OVX group $(\mathrm{P}<0.05, \mathrm{P}<0.01$ and $\mathrm{P}<0.01$, respectively). This occurred in a dose-dependent manner.

Effect of polydatin on $O P G$ and RANKL expression in ST2 cells. As shown in Fig. 6, polydatin (10, 20 and $40 \mu \mathrm{g} / \mathrm{ml})$ 

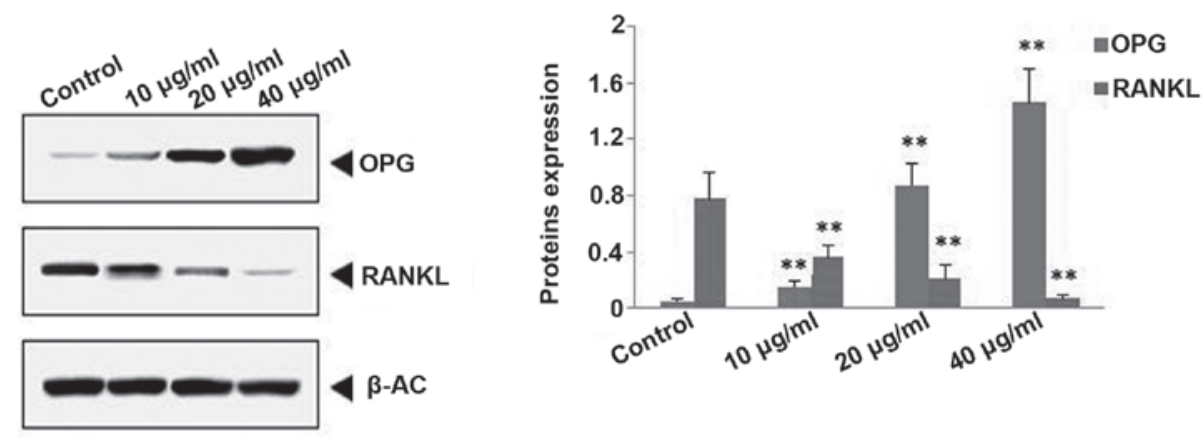

Figure 6. Effect of polydatin on OPG and RANKL in ST2 cells. ST2 cells were cultured with polydatin (10, 20 and $40 \mu \mathrm{g} / \mathrm{ml})$ for $48 \mathrm{~h}$. Protein expression of OPG and RANKL was measured by western blotting, and $\beta$-AC was used as an internal reference. Data are presented as the mean \pm standard deviation (n=4); ${ }^{* *} \mathrm{P}<0.01$, compared with control. OPG, osteoprotegerin; RANKL, receptor activators of nuclear factor- $\mathrm{kB}$ ligand; $\beta$-AC, $\beta$-actin.
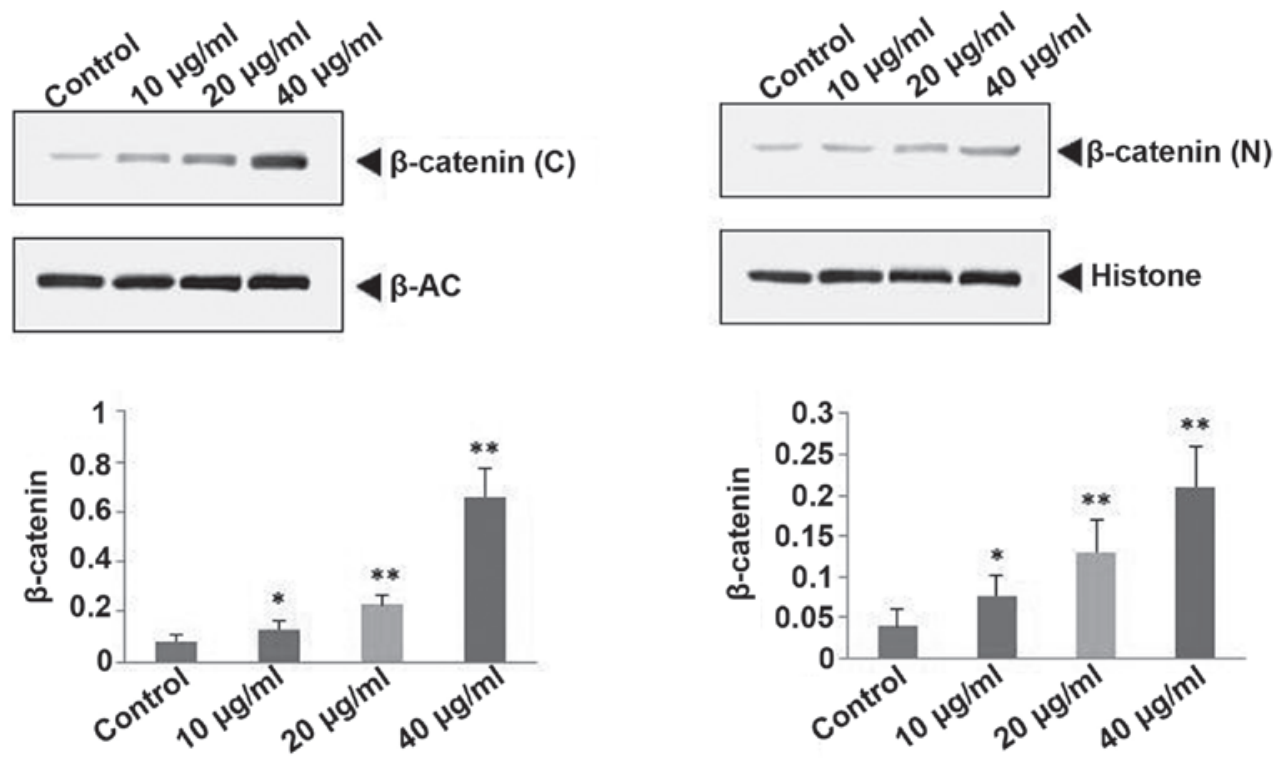

Figure 7. Effect of polydatin on the expression of $\beta$-catenin in ST2 cells. ST2 cells were cultured with polydatin (10, 20 and $40 \mu \mathrm{g} / \mathrm{ml})$ for $48 \mathrm{~h}$. Protein expression of $\beta$-catenin in the cytoplast and nucleus was measured by western blotting, and $\beta$-AC/histone was used as an internal reference. Data are presented as the mean \pm standard deviation $(n=4) ;{ }^{*} \mathrm{P}<0.05,{ }^{* *} \mathrm{P}<0.01$, compared with the control. $\beta$-AC, $\beta$-actin; $\mathrm{C}$, cytoplast; $\mathrm{N}$, nucleus.

significantly increased the protein expression of OPG in ST2 cells compared with control $(\mathrm{P}<0.01)$ in a concentration-dependent manner. Conversely, RANKL protein expression was downregulated by polydatin at a concentration of 10,20 and $40 \mu \mathrm{g} / \mathrm{ml}$ compared with the control $(\mathrm{P}<0.01)$, this occurred in a concentration-dependent manner. Consequently, the ratio of OPG/RANKL was upregulated following treatment with polydatin.

Effect of polydatin on $\beta$-catenin expression in ST2 cells. As shown in Fig. 7, 10, 20 and $40 \mu \mathrm{g} / \mathrm{ml}$ polydatin significantly upregulated the protein expression of OPG in the cytoplast and nucleus compared with the control $(\mathrm{P}<0.05, \mathrm{P}<0.01$ and $\mathrm{P}<0.01$, respectively), this occurred in a concentration-dependent manner.

\section{Discussion}

Increasing evidence has demonstrated that natural plant-derived constituents/extracts are promising potential resources for identifying effective candidate drugs. In addition, natural compounds are reported to have few side effects and may be candidates for treating various diseases (15-17). To the best of our knowledge, this study was the first to demonstrate that polydatin exhibited significant anti-osteoporotic activity on OVX-induced osteoporosis in mice.

Osteoporosis is associated with ovarian hormone deficiency following menopause, and is one of the most common reasons for bone loss. The ovariectomy-induced osteoporotic mouse model is a common and reliable animal model, which simulates the clinical symptoms of postmenopausal osteoporosis in women $(18,19)$. It is reported that ovariectomy resulted in body weight increases and uterus weight decreases (20). In the present study, polydatin was shown to increase the uterine index and decrease the body weight of mice that had undergone ovariectomy, suggesting that polydatin could improve the symptoms of osteoperosis. Bone loss can be reflected by the levels of calcium and phosphorus in serum (4). ALP is an important enzyme for bone remodeling, and the increase of ALP is another index for osteoporosis (21). The results showed 
significantly elevated calcium and phosphorus levels in the serum of ovariectomized mice, and decreased levels of ALP. These results indicated that polydatin possessed potential anti-osteoporotic activity for treating ovariectomized mice. ST2 cells are mesenchymal stem cells that have the ability to differentiate into osteoblast-like cells, and this cell line is commonly used to explore the mechanism of anti-osteoporotic drugs. The RANKL/OPG ratio is crucial in osteoclast formation and differentiation, and bone resorption. RANKL induces osteoclast differentiation via binding to RANK, and OPG can suppress osteoclastogenesis and bone resorption via blocking the communication between RANKL and RANK $(4,19)$. The present study showed that treatment with polydatin significantly decreased the expression of RANKL, and increased the expression of OPG, indicating that polydatin possessed the potential to inhibit bone loss and resorption. The Wnt/ $\beta$-catenin pathway is also important in the generation of osteoblasts, and bone development and remodeling. In addition, previous studies have demonstrated that upregulation of the $\mathrm{Wnt} / \beta$-catenin protein is a possible strategy for treating osteoporosis $(22,23)$. The results of the present study also demonstrated that the polydatin upregulates the expression of $\beta$-catenin proteins in the cytoplast and nucleus of ST2 cells.

In conclusion, the present study suggested that polydatin could alleviate the osteoporotic symptoms of OVX mice via upregulating OPG and $\beta$-catenin and downregulating RANKL. Furthermore, the present results may aid the development of polydatin as an effective drug to treat osteoporosis in the clinic.

\section{References}

1. Assessment of fracture risk and its application to screening for postmenopausal osteoporosis. Report of WHO study group. World Health Organ Tech Rep Ser 843: 1-129, 1994.

2. Rachner TD, Khosla S and Hofbauer LC: Osteoporosis: Now and the future. Lancet 377: 1276-1287, 2011

3. Bao L, Qin L, Liu L, Wu Y, Han T, Xue L and Zhang Q: Anthraquinone compounds from Morinda officinalis inhibit osteoclastic bone resorption in vitro. Chem Biol Interact 194: 97-105, 2011

4. LiH, Chen B,Pang G, Chen J, Xie J and Huang H: Anti-osteoporotic activity of puerarin 6'-O-xyloside on ovariectomized mice and its potential mechanism. Pharm Biol 54: 111-117, 2016.

5. Zhou ZX and Li LK: Research progress of traditional Chinese medicine treatment on osteoporosis. Drug Evaluation 10: 45-47, 2013.

6. Ma XQ, Zheng CJ, Zhang Y, Hu CL, Lin B, Fu XY, Han LY, $\mathrm{Xu}$ LS, Rahman K and Qin LP: Antiosteoporotic flavonoids from Podocarpium podocarpum. Phytochem Lett 6: 118-122, 2013.
7. Du J, Wei YJ, Peng C, Ran X, Zhang H, Jiang YP, Rahman K and Qin LP: Establishment of a luciferase assay-based screening system for detecting estrogen receptor agonists in plant extracts. Bone 49: 572-579, 2011.

8. Peng W, Qin R, Li X and Zhou H: Botany, phytochemistry, pharmacology, and potential application of Polygonum cuspidatum Sieb. et Zucc.: A review. J Ethnopharmacol 148: 729-745, 2013.

9. Lou T, Jiang W, Xu D, Chen T and Fu Y: Inhibitory effects of polydatin on lipopolysaccharide-stimulated RAW 264.7 cells. Inflammation 38: 1213-1220, 2015.

10. Wang HL, Gao JP, Han YL, Xu X, Wu R, Gao Y and Cui XH: Comparative studies of polydatin and resveratrol on mutual transformation and antioxidative effect in vivo. Phytomedicine 22: 553-559, 2015.

11. Shiyu S, Zhiyu L, Mao Y, Lin B, Lijia W, Tianbao Z, Jie C and Tingyu L: Polydatin up-regulates Clara cell secretory protein to suppress phospholipase A2 of lung induced by LPS in vivo and in vitro. BMC Cell Biol 12: 31, 2011.

12. Dong M, Ding W, Liao Y,Liu Y, Yan D, Zhang Y, Wang R, Zheng N, Liu S and Liu J: Polydatin prevents hypertrophy in phenylephrine induced neonatal mouse cardiomyocytes and pressure-overload mouse models. Eur J Pharmacol 746: 186-197, 2015.

13. Wu Y, Xue L, Du W, Huang B, Tang C, Liu C, Qiu H and Jiang Q: Polydatin restore endothelium-dependent relaxation in rat aorta rings impaired by high glucose: A novel insight into the PPAR $\beta$-NO signaling pathway. PLoS One 10: e0126249, 2015.

14. Kalu DN: The ovariectomized rat model of postmenopausal bone loss. Bone Miner 15: 175-191, 1991.

15. Kinghorn AD, Chin YW and Swanson SM: Discovery of natural product anticancer agents from biodiverse organisms. Curr Opin Drug Discov Devel 12: 189-196, 2009.

16. Bonifácio BV, dos Santos Ramos MA, da Silva PB and Bauab TM: Antimicrobial activity of natural products against Helicobacter pylori: A review. Ann Clin Microbiol Antimicrob 13: 54, 2014.

17. Cheng YC: Opportunities for traditional Chinese medicine to address unmet challenges in modern healthcare. J Tradit Complement Med 5: 2-4, 2015.

18. Riggs BL and Melton LJ III: Involutional osteoporosis. New Engl J Med 314: 1676-1686, 1986.

19. Xue L, Jiao L, Wang Y, Nie Y, Han T, Jiang Y, Rahman K, Zhang Q and Qin L: Effects and interaction of icariin, curculigoside, and berberine in er-xian decoction, a traditional Chinese medicinal formula, on osteoclastic bone resorption. Evid Based Complement Alternat Med 2012: 490843, 2012.

20. Li F, Yang XL, Yang YN, Guo C, Zhang C, Yang Z and Li P: Antiosteoporotic activity of echinacoside in ovariectomized rats. Phytomedicine 20: 549-557, 2013.

21. Yang L, Chen Q, Wang F and Zhang G: Antiosteoporotic compounds from seeds of Cuscuta chinensis. J Ethnopharmacol 135: 553-560, 2011.

22. Jeong BC, Kim TS, Kim HS, Lee SH and Choi Y: Transmembrane protein 64 reciprocally regulates osteoblast and adipocyte differentiation by modulating $\mathrm{Wnt} / \beta$-catenin signaling. Bone 78 : $165-173,2015$.

23. Tian J, Xu XJ, Shen L, Yang YP, Zhu R, Shuai B, Zhu XW, Li CG, $\mathrm{MaC}$ and Lv L: Association of serum Dkk-1 levels with $\beta$-catenin in patients with postmenopausal osteoporosis. J Huazhong Univ Sci Technolog Med Sci 35: 212-218, 2015. 